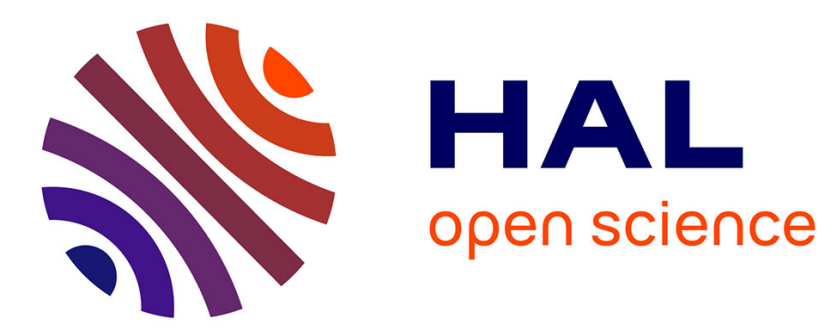

\title{
Delayed Sliding Mode Control
}

\author{
Denis Efimov, Andrey Polyakov, Leonid Fridman, Wilfrid Perruquetti, \\ Jean-Pierre Richard
}

\section{To cite this version:}

Denis Efimov, Andrey Polyakov, Leonid Fridman, Wilfrid Perruquetti, Jean-Pierre Richard. Delayed Sliding Mode Control. Automatica, 2016, 64, pp.37-43. 10.1016/j.automatica.2015.10.055 . hal01213703

\section{HAL Id: hal-01213703 https://inria.hal.science/hal-01213703}

Submitted on 8 Oct 2015

HAL is a multi-disciplinary open access archive for the deposit and dissemination of scientific research documents, whether they are published or not. The documents may come from teaching and research institutions in France or abroad, or from public or private research centers.
L'archive ouverte pluridisciplinaire HAL, est destinée au dépôt et à la diffusion de documents scientifiques de niveau recherche, publiés ou non, émanant des établissements d'enseignement et de recherche français ou étrangers, des laboratoires publics ou privés. 


\title{
Delayed Sliding Mode Control
}

\author{
Efimov D., Polyakov A., Fridman L., Perruquetti W., Richard J.-P.
}

\begin{abstract}
A new sliding mode control approach is introduced in this work with the dedicated mathematical tools. A timedelay modification/approximation of sign function is proposed, and it is shown that by substituting this new "sign" realization in the conventional sliding mode algorithms the main advantages of the sliding mode tools are preserved (like rejection of matched disturbances and hyper-exponential convergence ${ }^{1}$ ), while the chattering is reduced. These results are illustrated and confirmed by numerical simulations for the first order sliding mode control and the super-twisting algorithm.
\end{abstract}

\section{Index Terms}

Sliding mode, Time delay, Input-to-state stability

\section{INTRODUCTION}

The sliding mode control and estimation algorithms became very popular nowadays due to their strong advantages: finite time of convergence and compensation of matched disturbances [2], [3], [4]. Despite of that the sliding mode solutions have also several drawbacks mainly originated by the impossibility of a perfect practical realization of sliding motion. Among these shortages it is necessary to mention the chattering phenomenon, which is a high frequency oscillation of control signal when trajectories stay around the sliding surface. The appearance of chattering may physically destroy actuator and/or degrade the performance of transients [5], [6]. There are several approaches for chattering reduction [3]. One of the most popular deals with the high order sliding mode algorithms [7], [8], where the discontinuity, for example

$$
\operatorname{sign}[y(t)]=\left\{\begin{array}{ll}
\frac{y(t)}{|y(t)|}, & y(t) \neq 0 \\
0, & y(t)=0
\end{array},\right.
$$

with $y(t) \in \mathbb{R}$ and $|\cdot|$ is the absolute value, appears not in the control/estimated variable itself but in its derivative of higher order [2], [3]. However, since a discontinuity is present in the system closed loop, it may negatively influence the transients through the corresponding derivative (if this derivative has a physical meaning in the system). That is

D. Efimov, A. Polyakov, W. Perruquetti and J.-P. Richard are with Non-A team @ Inria, Parc Scientifique de la Haute Borne, 40 av. Halley, 59650 Villeneuve d'Ascq, France and CRIStAL (UMR-CNRS 9189), Ecole Centrale de Lille, BP 48, Cité Scientifique, 59651 Villeneuve-d'Ascq, France.

L. Fridman is with 4430 Institut für Regelungs- und Automatisierungstechnik 8010 Graz, Kopernikusgasse 24/II on leave of Departamento de Ingeniería de Control y Robótica, División de Ingeniería Eléctrica, Facultad de Ingeniería UNAM,Edificio T, Segundo piso Ciudad Universitaria D.F., México.

D. Efimov is with Department of Control Systems and Informatics, Saint Petersburg State University of Information Technologies Mechanics and Optics (ITMO), 49 Kronverkskiy av., 197101 Saint Petersburg, Russia.

This work was supported in part by ANR Grant CHASLIM (ANR-11-BS03-0007); by CNRS/CONACYT projects 222629, 209731; by CONACyT 261737; by the Government of Russian Federation (Grant 074-U01) and the Ministry of Education and Science of Russian Federation (Project 14.Z50.31.0031); by Programa de Apoyo a Proyectos de Investigacion e Innovacion Tecnologica (UNAM) 113613, DGAPA PASPA Program.

\footnotetext{
${ }^{1}$ i.e. the rate of convergence to the origin is much faster than any exponential [1].
} 
why frequently for practical realization of sliding mode algorithms different approximations of sign functions are used [9], [10], like for instance

$$
\hat{\operatorname{sign}}[y(t)]=\frac{y(t)}{\epsilon+|y(t)|}
$$

with some sufficiently small $\epsilon>0$, or see also [11] for interesting general sigmoid and dynamic approximations. Such a control based on approximated sign functions received the name of "continuous" sliding mode [12], [13], [14]. The main drawback of existent approximations is that a chattering reduction is achieved by a price of quality loss (appearance of static error in the presence of matched disturbances and, consequently, practical stability with an exponential rate of convergence [9], [15]). In the present work a development of the sliding mode control is presented that is based on a sign approximation using the time-delay framework, which guarantees the quality preservation (there is no static error in the presence of a matched disturbance and locally the speed of convergence is faster than any exponential).

Usually appearance of a delay in the system leads to performance degradation and complication of stability analysis. However in this work we are going to introduce delay in a proper way into the system, in order to make a modification of the sign function, proving certain performance in the system. The obtained approximation is very simple and can be easily implemented in control/estimation systems based on the sliding mode algorithms:

$$
\operatorname{sign}_{\tau}\left(y_{t}\right)=\frac{y(t)}{\max \{|y(t)|,|y(t-\tau)|\}},
$$

where $y_{t} \in C_{[-\tau, 0]}, y(t) \in \mathbb{R}$ is a variable whose sign has to be evaluated at time instant $t \in \mathbb{R}$ and $\tau>0$ is a fixed delay (design parameter). Obviously,

$$
\operatorname{sign}_{0}\left(y_{t}\right)=\operatorname{sign}[y(t)]
$$

In this work the new delayed sliding mode control framework will be substantiated, it is based on such an approximation and admits the following advantages:

- exact cancellation of matched disturbances is preserved despite of approximation;

- the control algorithms demonstrate hyper-exponential convergence to the origin;

- chattering reduction with respect to conventional sliding mode control.

The idea of chattering reduction, roughly speaking, is based on the fact that

$$
\left\{\phi \in C_{[-\tau, 0]}: \operatorname{sign}_{\tau}(\phi)=0\right\} \subset\left\{\phi \in C_{[-\tau, 0]}: \operatorname{sign}[\phi(0)]=0\right\} .
$$

The outline of this work is as follows. The preliminary definitions for time-delay systems are given in Section 2. The motivating example of the first order sliding mode control algorithm is considered in Section 3. An extension to high order sliding mode algorithms is presented in Section 4. Several examples are considered for illustration of the obtained results.

\section{Preliminaries}

Consider a functional differential equation of retarded type [16]:

$$
d x(t) / d t=f\left(x_{t}, d(t)\right), t \geq 0,
$$

where $x(t) \in \mathbb{R}^{n}$ and $x_{t} \in C_{[-\tau, 0]}$ is the state function, $x_{t}(s)=x(t+s),-\tau \leq s \leq 0$ (we denote by $C_{[-\tau, 0]}$, $0<\tau<+\infty$ the Banach space of continuous functions $\phi:[-\tau, 0] \rightarrow \mathbb{R}^{n}$ with the uniform norm $\|\phi\|=$ $\sup _{-\tau \leq \varsigma \leq 0}|\phi(\varsigma)|$, where $|\cdot|$ is the standard Euclidean norm); $d(t) \in \mathbb{R}^{m}$ is an essentially bounded measurable 
input, i.e. $\|d\|_{\infty}=e s s \sup _{t \geq 0}|d(t)|<+\infty ; f: C_{[-\tau, 0]} \times \mathbb{R}^{m} \rightarrow \mathbb{R}^{n}$ is a locally bounded functional, $f(\mathbf{0}, 0)=0$ where $\mathbf{0}(s)=0$ for all $-\tau \leq s \leq 0$. The representation (1) includes pointwise or distributed time-delay systems. We consider the system (1) with the initial functional condition $x_{0} \in C_{[-\tau, 0]}$.

A continuous function $\sigma: \mathbb{R}_{+} \rightarrow \mathbb{R}_{+}$belongs to class $\mathcal{K}$ if it is strictly increasing and $\sigma(0)=0$; it belongs to class $\mathcal{K}_{\infty}$ if it is also unbounded. A continuous function $\beta: \mathbb{R}_{+} \times \mathbb{R}_{+} \rightarrow \mathbb{R}_{+}$belongs to class $\mathcal{K} \mathcal{L}$ if $\beta(\cdot, r) \in \mathcal{K}$ and $\beta(r, \cdot)$ is a decreasing to zero for any fixed $r \in \mathbb{R}_{+}$.

\section{A. Discontinuous functional differential equations}

The dynamical systems subjected by a time-delay, whose models are described by functional differential equations, find their applications in different areas of science and technology [17]. Analysis of delay influence on the system stability is critical for many natural and human-developed systems [18], [16], [19]. The problem of stability investigation in time-delay systems is much more complicated than for ordinary differential equations since design of a Lyapunov-Krasovskii functional or a Lyapunov-Razumikhin function is a complex issue.

It is known from the theory of functional differential equations [16] that under the above assumptions the system (1) with a locally Lipschitz $f$ has a unique solution $x\left(t, x_{0}, d\right)$ satisfying the initial condition $x_{0}$ for the input $d(t)$, which is defined on some finite time interval $[-\tau, T)$ (we will use the notation $x(t)$ to reference $x\left(t, x_{0}, d\right)$ if the origin of $x_{0}$ and $d$ is clear from the context). If function $f(\phi, d) \equiv f_{0}(\phi(0), \phi(-\tau), d)$, where $f_{0}: \mathbb{R}^{2 n+m} \rightarrow \mathbb{R}^{n}$, and it is discontinuous with respect to $\phi(0)$ on a set $\mathcal{N} \subset \mathbb{R}^{n}$ of measure zero only and continuous with respect to $\phi(-\tau)$ and $d$, following [20], [21] (see also [22], [23] for a general definition and existence conditions of solutions for discontinuous functional differential equations) we will consider its multi-valued extension (define $B_{\varepsilon}(x)=\left\{y \in \mathbb{R}^{n}:|x-y| \leq \varepsilon\right\}$ as a closed ball of radius $\varepsilon>0$ around $\left.x \in \mathbb{R}^{n}\right)$ :

$$
F(\phi, d)=\bigcap_{\varepsilon>0} \overline{\operatorname{conv}}\left[f_{0}\left\{B_{\varepsilon}(\phi(0)) \backslash \mathcal{N}, \phi(-\tau), d\right\}\right],
$$

which is non-empty, compact, convex and upper semi-continuous [21] for any $d \in \mathbb{R}^{m}$. In particular, the multi-valued extension of $\operatorname{sign}_{\tau}\left(y_{t}\right)$ can be defined as follows:

$$
\overline{\operatorname{sign}}_{\tau}\left(y_{t}\right)=\left\{\begin{array}{ll}
{[-1,1],} & y(t)=y(t-\tau)=0 \\
\frac{y(t)}{\max \{|y(t)|,|y(t-\tau)|\}}, & \text { otherwise }
\end{array} .\right.
$$

In this case, instead of (1), the solutions of the following functional differential inclusion will be considered:

$$
d x(t) / d t \in F\left(x_{t}, d(t)\right), t \geq 0,
$$

and for any initial condition $x_{0} \in C_{[-\tau, 0]}$ the set of corresponding solutions of (2) initiated at $x_{0}$ for the input $d$ can be denoted as $\mathcal{S}\left(x_{0}, d\right)$.

Remark 1. Considering solutions of the system (1) for $f(\phi, d) \equiv f_{0}(\phi(0), \phi(-\tau), d)$ on the interval $[0, \tau]$ we can derive the Filippov differential inclusion for $\tilde{f}_{0}[t, x(t), d(t)]=f_{0}\left[x(t), x_{0}(t-\tau), d(t)\right]$ in the right-hand side. The obtained inclusion satisfies all conditions of existence theorem [20] implying existence of a solution locally. Having the solution defined on $[0, \tau]$ we can repeat the same considerations for $t \in[\tau, 2 \tau]$, etc. This method of steps allows solutions of (2) to be defined for $t>0$.

For a locally Lipschitz continuous function $V: \mathbb{R}^{n} \rightarrow \mathbb{R}_{+}$(where $\mathbb{R}_{+}=\{s \in \mathbb{R}: s \geq 0\}$ ) let us introduce the 
upper directional Dini derivative along the trajectories of (2):

$$
\begin{gathered}
D_{F\left(x_{t}, d\right)}^{+} V\left[x_{t}(0)\right]= \\
\sup _{v \in F\left(x_{t}, d\right)} \limsup _{h \rightarrow 0^{+}} \frac{V\left[x_{t}(0)+h v\right]-V\left[x_{t}(0)\right]}{h} .
\end{gathered}
$$

\section{B. Stability definitions}

Let $\Omega$ be an open neighborhood of the origin in $C_{[-\tau, 0]}$, and denote $\mathcal{D}$ set of all essentially bounded measurable input $d: \mathbb{R}_{+} \rightarrow \mathbb{R}^{m}$ with $\|d\|_{\infty}<D$ for some $0<D<+\infty$. Combining the results given in [24], [25], [22] we obtain the following stability notions.

Definition 1. The (trivial solution $x(t)=0$ of) system (2) for $d=0$ is said to be

(a) stable if there is $\sigma \in \mathcal{K}$ such that for any $x_{0} \in \Omega$ all solutions in $\mathcal{S}\left(x_{0}, 0\right)$ are defined for all $t \geq 0$ and for all $x\left(t, x_{0}, 0\right) \in \mathcal{S}\left(x_{0}, 0\right),\left|x\left(t, x_{0}, 0\right)\right| \leq \sigma\left(\left\|x_{0}\right\|\right)$ for all $t \geq 0$;

(b) asymptotically stable if it is stable and $\lim _{t \rightarrow+\infty}\left|x\left(t, x_{0}, 0\right)\right|=0$ for any $x_{0} \in \Omega$ and all $x\left(t, x_{0}, 0\right) \in$ $\mathcal{S}\left(x_{0}, 0\right)$

(c) finite-time stable if it is stable and for any $x_{0} \in \Omega$ there exists $0 \leq T^{x_{0}}<+\infty$ such that $x\left(t, x_{0}, 0\right)=0$ for all $t \geq T^{x_{0}}$ and all $x\left(t, x_{0}, 0\right) \in \mathcal{S}\left(x_{0}, 0\right)$. The functional $T_{0}\left(x_{0}\right)=\inf \left\{T^{x_{0}} \geq 0: x\left(t, x_{0}, 0\right)=0 \forall t \geq T^{x_{0}}\right\}$ is called the settling time of the system (1).

If $\Omega=C_{[-\tau, 0]}$, then the corresponding properties are called global stability/asymptotic stability/finite-time stability.

If the above properties hold for all $x\left(t, x_{0}, d\right) \in \mathcal{S}\left(x_{0}, d\right)$ with any $x_{0} \in \Omega$ and any $d \in \mathcal{D}$, then the corresponding properties are called uniform stability/asymptotic stability/finite-time stability.

For the forthcoming analysis we will need Lyapunov-Razumikhin theorem, which is given below (the variants for ordinary differential equations are given in [16], [18]).

Theorem 1. Let $\alpha_{1}, \alpha_{2} \in \mathcal{K}$ and $\eta: \mathbb{R}_{+} \rightarrow \mathbb{R}_{+}$be a continuous nondecreasing function. If there exists a Lipschitz continuous function $V: \mathbb{R}^{n} \rightarrow \mathbb{R}$ such that

$$
\alpha_{1}(|x|) \leq V(x) \leq \alpha_{2}(|x|), \forall x \in \mathbb{R}^{n}
$$

and the derivative of $V$ along a solution $x(t)$ of (2) satisfies

$$
\max _{\theta \in[-\tau, 0]} V[x(t+\theta)] \leq V[x(t)] \Rightarrow D_{F\left(x_{t}, 0\right)}^{+} V[x(t)] \leq-\eta(|x(t)|),
$$

then (2) is stable.

If, in addition, $\eta \in \mathcal{K}$ and there exists a continuous nondecreasing function $p(s)>s$ for $s>0$ such that the condition (3) is strengthened to

$$
\max _{\theta \in[-\tau, 0]} V[x(t+\theta)] \leq p\{V[x(t)]\} \Rightarrow D_{F\left(x_{t}, 0\right)}^{+} V[x(t)] \leq-\eta(|x(t)|),
$$

then (2) is asymptotically stable.

If in addition $\alpha_{1} \in \mathcal{K}_{\infty}$, then (2) is globally asymptotically stable. If the above properties are satisfied not only for $d=0$ but for any $d \in \mathcal{D}$, then (2) is uniformly stablelasymptotically stable.

Since the conditions of Theorem 1 are formulated for a Lyapunov function $V$, then the proof is the same for a differential equation or inclusion (it is omitted in this note). 
Similarly, in order to analyze robustness of functional differential inclusions we will use an extension of the input-to-state stability (ISS) framework proposed in [26], [25].

Definition 2. The system (2) is called ISS, if there exist $\beta \in \mathcal{K} \mathcal{L}$ and $\gamma \in \mathcal{K}$ such that for all $t \geq 0$ it holds

$$
\left|x\left(t, x_{0}, d\right)\right| \leq \beta\left(\left\|x_{0}\right\|, t\right)+\gamma\left(\|d\|_{\infty}\right)
$$

for all $x_{0} \in C_{[-\tau, 0]}$, all essentially bounded measurable inputs $d$ and all $x\left(t, x_{0}, d\right) \in \mathcal{S}\left(x_{0}, d\right)$.

Definition 3. A locally Lipschitz function $V: \mathbb{R}^{n} \rightarrow \mathbb{R}_{+}$is called ISS Lyapunov-Razumikhin function for (2), if there exist $\alpha_{1}, \alpha_{2} \in \mathcal{K}_{\infty}$ and $\chi_{\tau}, \chi_{d}, \alpha \in \mathcal{K}$ such that the following conditions hold:

$$
\begin{gathered}
\alpha_{1}(|x|) \leq V(x) \leq \alpha_{2}(|x|) \quad \forall x \in \mathbb{R}^{n} ; \\
V(x(t)) \geq \chi_{\tau}\left[\sup _{\theta \in[-\tau, 0]} V\{x(t+\theta)\}\right]+\chi_{d}(|d(t)|) \\
\Rightarrow D_{F\left(x_{t}, d\right)}^{+} V[x(t)] \leq-\alpha(V[x(t)]),
\end{gathered}
$$

for all solutions of (2) $x\left(t, x_{0}, d\right) \in \mathcal{S}\left(x_{0}, d\right)$ for all $x_{0} \in C_{[-\tau, 0]}$ and all essentially bounded measurable inputs $d$.

Theorem 2. [26] If there exists an ISS Lyapunov-Razumikhin function $V$ for (2) and $\chi_{\tau}(s)<s$ for all $s>0$, then the functional differential inclusion (2) is ISS from $d$ to $x$ with the gain $\gamma(s)=\alpha_{1}^{-1} \circ \chi_{d}(s)$.

\section{APPROXIMATION OF THE FIRST ORDER SLIDING MODE ALGORITHM}

\section{A. The rate of convergence}

First of all, let us consider the system

$$
\dot{x}(t)=-k \operatorname{sign}_{\tau}\left(x_{t}\right)
$$

for $x_{0} \in C_{[-\tau, 0]}$ with $\tau>0$ and $k>0$, in order to evaluate the rate of convergence in a system with the introduced approximation of the sign function, in this case $F\left(x_{t}, 0\right)=-k \overline{\operatorname{sign}}_{\tau}\left(x_{t}\right)$. First of all note that for (4) the origin $\left(\mathbf{0} \in C_{[-\tau, 0]}\right.$ with $\mathbf{0}(s)=0$ for all $\left.s \in[-\tau, 0]\right)$ is the equilibrium: once $x\left(t^{\prime}\right)=0$ for some $t^{\prime} \geq 0$, then $x(t)=0$ for all $t \geq t^{\prime}$. For any $x_{0} \in C_{[-\tau, 0]}$ consider a Lyapunov function candidate $V[x(t)]=\frac{1}{2} x^{2}(t)$, for (4) it has the derivative

$$
\begin{gathered}
D_{F\left(x_{t}, 0\right)}^{+} V[x(t)]=-k \frac{x^{2}(t)}{\max \{|x(t)|,|x(t-\tau)|\}} \\
=-k \alpha(t)|x(t)| \leq 0, \alpha(t)=\left\{\begin{array}{ll}
1, & |x(t)| \geq|x(t-\tau)| \\
\frac{|x(t)|}{|x(t-\tau)|}, & |x(t)|<|x(t-\tau)|
\end{array} .\right.
\end{gathered}
$$

Let $\max _{\theta \in[-\tau, 0]} V[x(t+\theta)] \leq p V[x(t)]$ for some $p>1$, then by definition of $V[x(t)]$ we obtain $|x(t-\tau)| \leq$ $\sqrt{p}|x(t)|$, or equivalently, $\alpha(t) \in\left[p^{-0.5}, 1\right]$, and $D_{F\left(x_{t}, 0\right)}^{+} V[x(t)] \leq-k p^{-0.5}|x(t)|$ that by Theorem 1 implies global asymptotically stability for (4).

Moreover, $\dot{V}(t)=D_{F\left(x_{t}, 0\right)}^{+} V[x(t)]<0$ wherein $x(t) \neq 0$, thus $V(t)$ and $|x(t)|$ are strictly decreasing. Therefore, $\operatorname{sign}[x(t)]=\operatorname{sign}[x(t-\tau)]$ and $|x(t)|<|x(t-\tau)|, x(t-\tau) \neq 0$ for all $t \geq \tau$, then

$$
x(t)=x(t-\tau)-\int_{t-\tau}^{t} k \frac{x(s)}{\max \{|x(s)|,|x(s-\tau)|\}} d s
$$




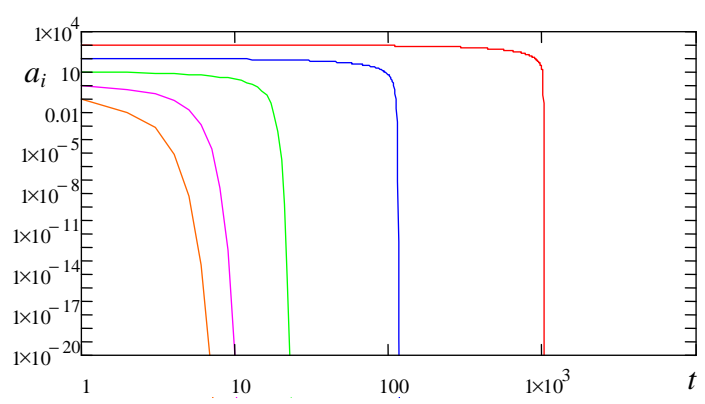

Figure 1. The results of the sequence (5) calculation

and for $t \geq 2 \tau$

$$
\begin{aligned}
|x(t)| & =|x(t-\tau)|-\int_{t-\tau}^{t} k \frac{|x(s)|}{|x(s-\tau)|} d s \\
& \leq|x(t-\tau)|-\frac{|x(t)|}{|x(t-2 \tau)|} \int_{t-\tau}^{t} k d s \\
& =|x(t-\tau)|-k \tau \frac{|x(t)|}{|x(t-2 \tau)|} .
\end{aligned}
$$

Thus for $t \geq 2 \tau$ we obtain

$$
|x(t)| \leq \frac{|x(t-\tau)||x(t-2 \tau)|}{|x(t-2 \tau)|+k \tau}
$$

and consider the sequence of $a_{i}=|x(i \tau)|$ for $i \geq 1$, then

$$
a_{i+1} \leq \frac{a_{i} a_{i-1}}{a_{i-1}+k \tau}, i \geq 1 .
$$

The rate of convergence of this sequence is polynomial, and for small amplitudes of $x(t)$, the state of (4) converges to zero with a hyper-exponential speed. To confirm this conclusion, the results of this sequence calculation for different initial values of $a_{0}=a_{1}$ and for $k \tau=1$ are shown in Fig. 1 in logarithmic scale.

Thus the following result has been proven.

Lemma 1. The system (4) is globally asymptotically stable, and it has a hyper-exponential rate of convergence.

Consequently, the proven time of convergence in (4) is not finite, and by this the convergence of (4) is slower than in the original system

$$
\dot{x}(t)=-k \operatorname{sign}[x(t)]
$$

but anyway it is much faster than an exponential rate, which can be obtained using standard approximations (rather restricted possibilities of finite-time convergence for time-delay systems are discussed in [27]).

Another consequence of this result is that in (4) there is no chattering of the right-hand side (the right hand-side of (4) is discontinuous for $x(t)=x(t-\tau)=0$, but that state is reached asymptotically). In other words, for all solutions of (4) with nonzero initial conditions, i.e. $x(s) \neq 0$ for all $s \in[-\tau, 0]$, the value of provided approximation for control law $\operatorname{sign}_{\tau}\left(x_{t}\right)$ is continuous for $t \in[0,+\infty)$ (this fact can also be seen in Fig. 2).

Of course, appearance of chattering is also dependent on the method of calculation of solutions and discretization step. Advantage of (4) is that it admits a simple discretization by explicit

$$
x\left(t_{i+1}\right)=x\left(t_{i}\right)-\frac{k h x\left(t_{i}\right)}{\max \left\{\left|x\left(t_{i}\right)\right|,\left|x\left(t_{i}-\tau\right)\right|\right\}}
$$



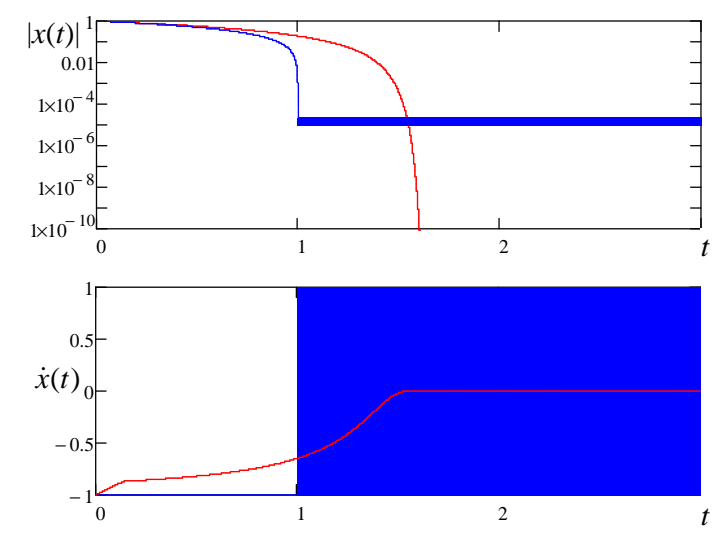

Figure 2. Results of simulation for (4) and (6)

or implicit (see also [28])

$$
x\left(t_{i}\right)=\left(1+\frac{k h}{\max \left\{\left|x\left(t_{i+1}\right)\right|,\left|x\left(t_{i}-\tau\right)\right|\right\}}\right) x\left(t_{i+1}\right)
$$

Euler method, where $t_{i}=h i, i \geq 0$ are the discrete times and $h$ is the discretization step. Since expression in the brackets is always bigger than 1 , for $t_{i} \geq \tau$ the implicit Euler discretization becomes explicit:

$$
x\left(t_{i+1}\right)=\left(1+\frac{k h}{\left|x\left(t_{i}-\tau\right)\right|}\right)^{-1} x\left(t_{i}\right),
$$

which is clearly converging for any $h>0$ (that is not true for the explicit Euler discretization). For an illustration, the results of simulation of (4) and (6) with $h=3 \times 10^{-5}$ are given in Fig. 2 for $x(s)=1, s \in[-\tau, 0]$ and $k=1$ and $\tau=0.15$. The red lines represent the variables $|x(t)|$ and $\dot{x}(t)$ generated by the system (4) (explicit and implicit discretizations give the same results), the blue lines correspond to the variables of the system (6).

\section{B. Uniform and robust stability/convergence}

Now let us consider the perturbed version of (4):

$$
\dot{x}(t)=-k \operatorname{sign}_{\tau}\left(x_{t}+d_{1 t}\right)+d_{2}(t)
$$

where $d_{1}(t) \in \mathbb{R}$ and $d_{2}(t) \in \mathbb{R}$ represent the measurement noise and the additive disturbance, respectively (they are locally bounded measurable functions of time). As usual in the sliding mode theory, we will be interested in the case when $\left\|d_{2}\right\|_{\infty}<D$ and $k>D$, in this case

$$
\begin{aligned}
\dot{x}(t) & \in F\left(x_{t}, d_{1 t}\right), \\
F\left(x_{t}, d_{t}\right) & =-k \overline{\operatorname{sign}}_{\tau}\left(x_{t}+d_{t}\right)+[-D, D] .
\end{aligned}
$$

Lemma 2. Let $\frac{\sqrt{2 b}-1}{\sqrt{2 b} p^{-1}+1} k>D$ for some $0<p<1$ and $b>0.5$. Then (8) is ISS from $d_{1}(t)$ to $x(t)$ with the gain $\gamma(s)=2 \sqrt{2 b} s$.

Proof. Consider for (8) a Lyapunov function candidate $V[x(t)]=\frac{1}{2} x^{2}(t)$ :

$$
D_{F\left(x_{t}, d_{1 t}\right)}^{+} V[x(t)] \leq-k x(t) \overline{\operatorname{sign}}_{\tau}\left(x_{t}+d_{1 t}\right)+|x(t)| D .
$$

Under conditions of the lemma, there exists an $\epsilon>0$ such that $\frac{\sqrt{2 b}-1}{\sqrt{2 b} p^{-1}+1} k \geq D+\epsilon$, where $0<p<1$ and $b>0.5$, 
and in order to apply Theorem 2 assume that the following restriction is satisfied on trajectories of (8):

$$
\begin{aligned}
V[x(t)] & \geq b\left|d_{1}(t)\right|^{2}+b\left|d_{1}(t-\tau)\right|^{2}+p^{2} V[x(t-\tau)] \\
& \geq \max \left\{b\left|d_{1}(t)\right|^{2}, b\left|d_{1}(t-\tau)\right|^{2}, p^{2} V[x(t-\tau)]\right\}
\end{aligned}
$$

then

$$
\left.|x(t)| \geq \max \left\{\sqrt{2 b}\left|d_{1}(t)\right|, \sqrt{2 b}\left|d_{1}(t-\tau)\right|, p|x(t-\tau)|\right]\right\}
$$

and it is necessary to prove that $D_{F\left(x_{t}, d_{1 t}\right)}^{+} V[x(t)]<0$ under (9). In this case $\operatorname{sign}\left[x(t)+d_{1}(t)\right]=\operatorname{sign}[x(t)]$ and

$$
D_{F\left(x_{t}, d_{1 t}\right)}^{+} V[x(t)] \leq-k|x(t)| g\left(x_{t}, d_{1 t}\right)+|x(t)| D,
$$

where

$$
g\left(x_{t}, d_{1 t}\right)=\frac{\left|x(t)+d_{1}(t)\right|}{\max \left\{\left|x(t)+d_{1}(t)\right|,\left|x(t-\tau)+d_{1}(t-\tau)\right|\right\}} .
$$

Obviously, $g\left(x_{t}, d_{1 t}\right)=1$ if $\left|x(t)+d_{1}(t)\right| \geq\left|x(t-\tau)+d_{1}(t-\tau)\right|$. Consider the case $\left|x(t)+d_{1}(t)\right|<\mid x(t-\tau)+$ $d_{1}(t-\tau) \mid$, then

$$
g\left(x_{t}, d_{1 t}\right)=\frac{\left|x(t)+d_{1}(t)\right|}{\left|x(t-\tau)+d_{1}(t-\tau)\right|}
$$

and under the imposed restrictions (9), $\left|x(t)+d_{1}(t)\right| \geq\left(1-\frac{1}{\sqrt{2 b}}\right)|x(t)|$ with $\left|x(t-\tau)+d_{1}(t-\tau)\right| \leq\left[p^{-1}+\frac{1}{\sqrt{2 b}}\right]|x(t)|$, hence $g\left(x_{t}, d_{1 t}\right) \geq \frac{\sqrt{2 b}-1}{\sqrt{2 b} p^{-1}+1}$ in this case. Therefore, since $0<\frac{\sqrt{2 b}-1}{\sqrt{2 b} p^{-1}+1} \leq p<1$ for any $0<p<1$ and $b>0.5$, the following relation has been proven under (9):

$$
\begin{aligned}
D_{F\left(x_{t}, d_{1 t}\right)}^{+} V[x(t)] & \leq\left(D-k \frac{\sqrt{2 b}-1}{\sqrt{2 b} p^{-1}+1}\right)|x(t)| \\
& \leq-\epsilon|x(t)|=-\epsilon \sqrt{2 V[x(t)]} .
\end{aligned}
$$

Thus, $V$ is an ISS Lyapunov-Razumikhin function for (8) with respect to an extended input $\left[d_{1}(t) d_{1}(t-\tau)\right]^{T}$ for $\alpha_{1}(s)=\alpha_{2}(s)=0.5 s^{2}, \alpha(s)=\epsilon \sqrt{2 s}, \chi_{\tau}(s)=p^{2} s$ and $\chi_{d}(s)=b s^{2}$. Since $\chi_{\tau}(s)<s$ for all $s>0$, then according to Theorem 2 the functional differential inclusion (8) is ISS from $\left[d_{1}(t) d_{1}(t-\tau)\right]^{T}$ to $x(t)$ with the gain $\gamma(s)=\alpha_{1}^{-1} \circ \chi_{d}(s)$. Taking in mind definition of ISS property, it implies that (8) is ISS from $d_{1}(t)$ to $x(t)$ with the gain $\gamma(s)=\alpha_{1}^{-1} \circ \chi_{d}(2 s)$.

For (7) the result of Lemma 2 implies that for proposed $k>D$ the system is ISS with respect to measurement noise $d_{1}$ uniformly on matched disturbances $\left\|d_{2}\right\|_{\infty}<D$. Therefore, if $d_{1}=0$ (there is no noise), then any disturbance $\left\|d_{2}\right\|_{\infty}<D$ will be rejected in (7). The parameter $b$ defines the gain $\gamma$ of the system (7) with respect to measurement noise $d_{1}$ and, to this end, it determines the value of $k$.

The results of simulation with the discretization step $h=3 \times 10^{-5}$ in the Euler method for (7) and the conventional sliding mode system

$$
\dot{x}(t)=-k \operatorname{sign}\left[x(t)+d_{1}(t)\right]+d_{2}(t)
$$

are shown in figures 3 and 4 for $\tau=0.15$ and $k=1$ (in this case $b=10^{3}, p=0.96$ and $\epsilon=10^{-2}$ for the given $D=0.9$ ). In the top of Fig. 3 the variable $x(t)$ is shown for both (7) (red line) and (10) (blue line), for $d_{1}(t)=0$ and $d_{2}(t)=0.3[\sin (15 t)+\sin (\pi t)+1]$. At the bottom of the figure, on the left the variable $u(t)=\operatorname{sign}_{\tau}\left(x_{t}+d_{1 t}\right)$ for (7) (red line) and the signal $-d_{2}(t)$ (green dash line) are plotted (the variable $u(t)$ represents a kind of "control" approximation in a sliding mode control system), on the right the variable $u(t)=\operatorname{sign}_{\tau}\left(x_{t}+d_{1 t}\right)$ for (7) and the variable $u(t)=\operatorname{sign}\left[x(t)+d_{1}(t)\right]$ for (10) are shown (the latter one presents a pure chattering after approximately 

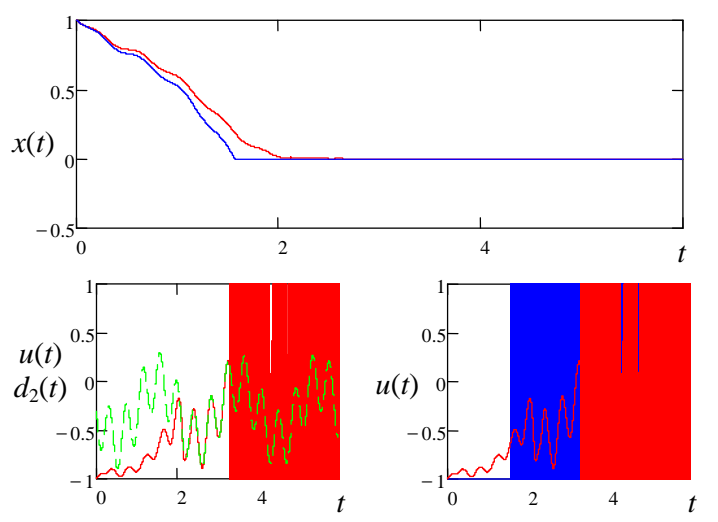

Figure 3. Trajectories of (7) (red) and (10) (blue) for $d_{1}=0$

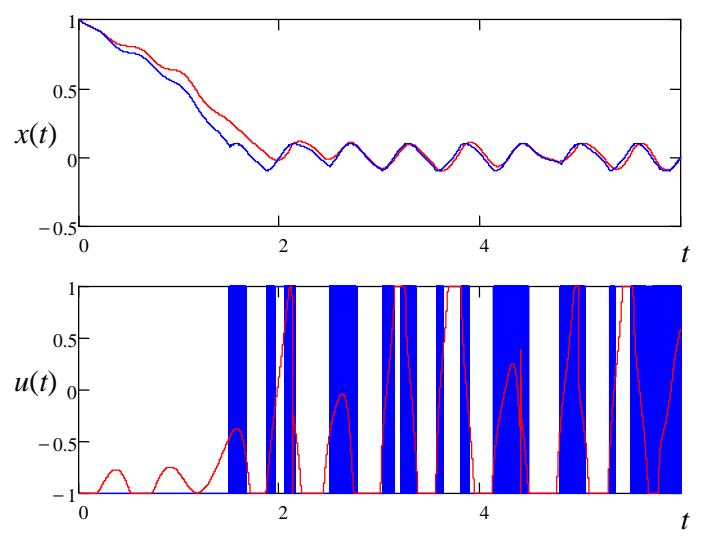

Figure 4. Trajectories of (7) (red) and (10) (blue) with $d_{1}(t)=0.1 \sin (11 t)$

1.5 second of time). As we can conclude, the system (7) has less chattering. In Fig. 4 the results of simulation are shown for $d_{1}(t)=0.1 \sin (11 t)$ and the same $d_{2}(t)$, the variables $x(t)$ and $u(t)$ are shown for (7) (red lines) and (10) (blue lines). In this case, again, the variable $x(t)$ has a similar behavior for (7) and (10), but $u(t)$ contains less chattering for (7).

\section{APPROXIMATION OF HIGH ORDER SLIDING MODE ALGORITHMS}

The main idea of the proof of Lemma 2 has the following interpretation: if for $\operatorname{sign}\left[x(t)+d_{1}(t)\right]$ the sliding mode system has an ISS Lyapunov function $V$, and $\operatorname{since} \operatorname{sign}_{\tau}\left(x_{t}+d_{1 t}\right)$ can be reduced to $\operatorname{sign}\left[x(t)+d_{1}(t)\right]$ under the Lyapunov-Razumikhin arguments, then $V$ is an ISS Lyapunov-Razumikhin function for the system with $\operatorname{sign}_{\tau}\left(x_{t}+d_{1 t}\right)$. Thus a simple extension for high order sliding mode systems can be obtained.

Consider a conventional sliding mode system:

$$
\dot{x}(t) \in G(x(t), \overline{\operatorname{sign}}\{s[x(t)]\}, d(t)),
$$

where $x(t) \in \mathbb{R}^{n}$ is the state, $d(t) \in \mathbb{R}^{m}$ is the disturbance, $G: \mathbb{R}^{n+m+k} \rightarrow \mathbb{R}^{n}$ and $s: \mathbb{R}^{n} \rightarrow \mathbb{R}^{k}$ are continuous functions, $s(t)=s[x(t)] \in \mathbb{R}^{k}$ is the sliding surface variable (application of the sign function to a vector argument is understood elementwise).

Assumption 1. Let the system (11) be ISS from $d$ to $x$ and there exist an ISS Lyapunov function $V: \mathbb{R}^{n} \rightarrow \mathbb{R}_{+}$ 
such that $\forall x \in \mathbb{R}^{n}$ and $\forall d \in \mathbb{R}^{m}$

$$
\begin{gathered}
\alpha_{1}(|x|) \leq V(x) \leq \alpha_{2}(|x|) ; \\
V(x(t)) \geq \chi_{d}(|d(t)|) \Rightarrow \\
D_{G(x(t), \overline{\operatorname{sign}}\{s[x(t)]\}, d)}^{+} V[x(t)] \leq-\alpha(V[x(t)])
\end{gathered}
$$

for some $\alpha_{1}, \alpha_{2} \in \mathcal{K}_{\infty}$ and $\chi_{d}, \alpha \in \mathcal{K}$.

Let us introduce another time-delayed modification of the sign function for the high order case:

$$
\overline{\operatorname{sign}}_{\tau}\left(x_{t}\right)= \begin{cases}\frac{s[x(t)]}{\max \{|s[x(t)],| s[x(t-\tau)] \mid\}}, & V[x(t)]<p V[x(t-\tau)] \\ & \text { and }|s[x(t)]| \neq 0 \\ \frac{s[x(t)],}{\mid s[x(t)],} & V[x(t)] \geq p V[x(t-\tau)] \\ & \text { and }|s[x(t)]| \neq 0 \\ {[-1,1]^{k},} & |s[x(t)]|=0\end{cases}
$$

for some constant $0<p<1$, where $V$ has been introduced in Assumption 1. As before, $\overline{\operatorname{sign}}_{0}\left(x_{t}\right)=\overline{\operatorname{sign}}[x(t)]$.

Theorem 3. Let Assumption 1 be satisfied for the system (11). Then for any $0<\tau<+\infty$ the system

$$
\dot{x}(t) \in G\left(x(t), \overline{\operatorname{sign}}_{\tau}\left(x_{t}\right), d(t)\right),
$$

is also ISS from $d$ to $x$.

Proof. In order to apply Theorem 2, let for $0<p<1$ and $V(x)$ from Assumption 1 the following relation be satisfied for the system (12):

$$
V(x(t)) \geq p V(x(t-\tau))+\chi_{d}(|d(t)|),
$$

then

$$
V(x(t)) \geq \max \left\{p V(x(t-\tau)), \chi_{d}(|d(t)|)\right\}
$$

and according to the definition of $\overline{\operatorname{sign}}_{\tau}\left(x_{t}\right)$ we have $\overline{\operatorname{sign}}_{\tau}\left(x_{t}\right)=\overline{\operatorname{sign}}\{s[x(t)]\}$ and

$$
D_{G\left(x(t), \overline{\operatorname{sign}}_{\tau}\left(x_{t}\right), d\right)}^{+} V[x(t)] \leq-\alpha(V[x(t)]) .
$$

Thus $V$ is an ISS Lyapunov-Razumikhin function for (12) for $\chi_{\tau}(s)=p s$. Since $\chi_{\tau}(s)<s$ for all $s>0$, then according to Theorem 2 the functional differential inclusion (12) is ISS from $d$ to $x$ with the gain $\gamma(s)=$ $\alpha_{1}^{-1} \circ \chi_{d}(s)$.

For an illustration of the result of this theorem consider the super-twisting algorithm:

$$
\begin{aligned}
& \dot{x}_{1}(t)=-k_{1} \sqrt{\left|x_{1}(t)\right|} \operatorname{sign}\left[x_{1}(t)\right]+x_{2}(t), \\
& \dot{x}_{2}(t)=-k_{2} \operatorname{sign}\left[x_{1}(t)\right]+d(t),
\end{aligned}
$$

where $0<k_{1}<k_{2}$ are parameters,

$$
G(x, \sigma, d)=\left[\begin{array}{c}
-k_{1} \sqrt{\left|x_{1}\right|} \operatorname{sign}\left[x_{1}\right]+x_{2} \\
-k_{2} \sigma+d
\end{array}\right] .
$$

As a Lyapunov function candidate take one from [29] (the Lyapunov function proposed in [30] also can be used) 


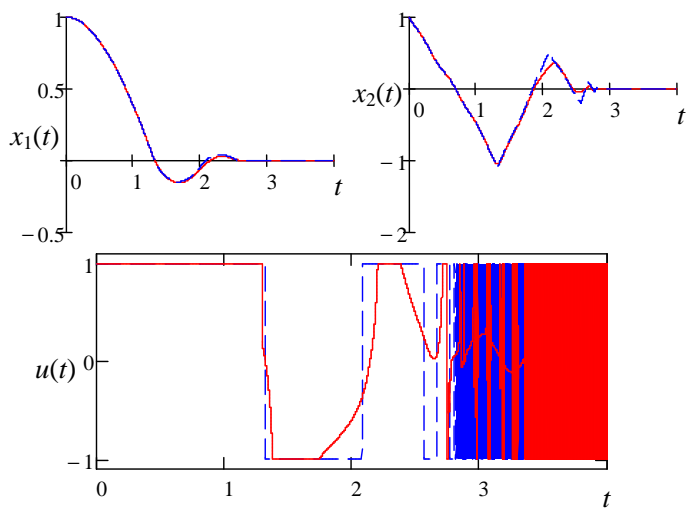

Figure 5. Results of simulation for super-twisting algorithm

and $s(x)=x_{1}$. The results of simulation for $k_{1}=1, k_{2}=2, \tau=0.1$ and $d(t)=0.3[\sin (15 t)+\sin (\pi t)+1]$ with the discretization step $h=4 \times 10^{-5}$ in the Euler method are shown in Fig. 5. The red solid lines represent the variables of time-delayed algorithm, the blue dash lines correspond to the variables of (13), the variable $u(t)$ in both cases demonstrate the function $\operatorname{sign}(\cdot)$ behavior and its approximation. As we can conclude from this numerical experiment, the higher order sliding mode algorithm behavior is quite nice and smooth (see the variables $x_{1}(t)$ and $x_{2}(t)$ ), but even in this case a chattering reduction is achieved by a slight decrease of the convergence rate using the new continuous delayed sliding mode algorithm.

\section{Conclusions}

In this note the Delayed Sliding Mode control has been presented, which demonstrates how by a delay introduction in the closed-loop of a nonlinear system it is possible to improve the quality of steady-state behavior. An approximation of the sign function has been given based on delayed values of the processed variable, which ensures chattering reduction for the sliding mode control and estimation algorithms. The delay has been introduced in a way that under the Lyapunov-Razumikhin conditions the system is reduced to its original non-delayed form, then its stability can be easily concluded. Efficiency of the proposed approach has been demonstrated on examples: the chattering can be avoided (see Fig. 2) or tolerated (see figures 3-5) by a slight decreasing of the convergence rate, in addition, the matched disturbances are compensated. Influence of discrete-time realization on the obtained in continuous time properties, tuning rules and asymptotic performance analysis are directions of future research.

\section{REFERENCES}

[1] A. Polyakov, D. Efimov, W. Perruquetti, and J.-P. Richard, "Implicit lyapunov-krasovski functionals for time delay systems," in Proc. 53rd IEEE CDC, (LA), 2014.

[2] W. Perruquetti and J. P. Barbot, Sliding Mode Control in Engineering. Marcel Dekker Hardcover, 2002.

[3] L. Fridman, Sliding Mode Enforcement after 1990: Main Results and Some Open Problems, vol. 412 of LNCIS, pp. 3-57. Springer Verlag Berlin Heidelberg, 2011.

[4] I. Boiko, Discontinuous Control Systems: Frequency-Domain Analysis and Design. Birkhauser, 2009.

[5] V. I. Utkin, Sliding Modes in Control Optimization. Berlin: Springer-Verlag, 1992.

[6] A. Levant, "Chattering analysis," IEEE Transactions on Automatic Control, vol. 55, no. 6, pp. 1380-1389, 2010.

[7] A. Levant, "Sliding order and sliding accuracy in sliding mode control," International Journal of Control, vol. 58, no. 6, pp. 1247-1263, 1993.

[8] C. Edwards and Y. Shtessel, "Continuous higher order sliding mode control based on adaptive disturbance compensation," in 13th International Workshop on Variable Structure Systems (VSS), pp. 1-5, June 2014.

[9] G. Ambrosino, G. Celentano, and F. Garofalo, "Robust model tracking control for a class of nonlinear plant," IEEE Trans. on Automatic Control, vol. AC-30, no. 3, pp. 275-279, 1985. 
[10] J. Burton and A. Zinober, “Continuous approximation of variable structure control," International Journal of Systems Science, vol. 17, no. 6, pp. $875-885,1986$.

[11] C. Canudas-de Wit and W. Perruquetti, "Smoothing strategies for high-gain control," in Proc. IFAC Latin-American Conference on Automatic Control, (Mexico), 2002.

[12] H. Khalil, Nonlinear Systems. Upper Saddle River, New Jersey: Prentice Hall, 3rd ed., 2002.

[13] H. Oza, Y. Orlov, S. Spurgeon, Y. Aoustin, and C. Chevallereau, "Continuous second order sliding mode based robust finite time tracking of a fully actuated biped robot," in Proc. European Control Conference (ECC), pp. 2600-2605, June 2014.

[14] Y. B. Shtessel, I. A. Shkolnikov, and M. D. Brown, "An asymptotic second-order smooth sliding mode control," Asian Journal of Control, vol. 5, pp. 498-504, 2003.

[15] F. Esfandiari and H. Khalil, "Stability analysis of a continuous implementation of variable structure control," IEEE Trans. on Automatic Control, vol. 36, no. 5, pp. 616-620, 1991.

[16] V. Kolmanovsky and V. Nosov, Stability of functional differential equations. San Diego: CA: Academic, 1986.

[17] J. Chiasson and J. Loiseau, eds., Applications of Time Delay Systems, vol. 352 of Lecture Notes in Control and Information Sciences. Springer, 2007.

[18] K. Gu, K. Kharitonov, and J. Chen, Stability of Time-Delay Systems. Control Engineering, Boston: Birkhäuser, 2003.

[19] J.-P. Richard, "Time-delay systems: an overview of some recent advances and open problems," Automatica, vol. 39, pp. 1667-1694, 2003.

[20] A. F. Filipov, Differential Equations with Discontinuous Righthand Sides. Kluwer Academic Publishers, 1988.

[21] W. Heemels and S. Weiland, "Input-to-state stability and interconnections of dicontinuous dynamical systems," Automatica, vol. 44, no. 12, pp. 3079-3086, 2008.

[22] V. Kolmanovskii and A. Myshkis, Introduction to the theory and applications of functional differential equations. Dordrecht, The Netherlands: Kluwer, 1999.

[23] A. Surkov, “On functional-differential equations with discontinuous right-hand side,” Differential Equations, vol. 44, no. 2, pp. 278-281, 2008.

[24] E. Moulay, M. Dambrine, N. Yeganefar, and W. Perruquetti, "Finite-time stability and stabilization of time-delay systems," Systems Control Lett., vol. 57, pp. 561-566, 2008.

[25] P. Pepe and H. Ito, "On saturation, discontinuities, and delays, in iiss and iss feedback control redesign," IEEE Trans. Automat. Control, vol. 57, no. 5, pp. 1125-1140, 2012.

[26] A. R. Teel, "Connections between Razumikhin-type theorems and the ISS nonlinear small gain theorem," IEEE Trans. Automat. Control, vol. 43, no. 7, pp. 960-964, 1998.

[27] D. Efimov, A. Polyakov, E. Fridman, W. Perruquetti, and J.-P. Richard, "Comments on finite-time stability of time-delay systems," Automatica, vol. 50, no. 7, pp. 1944-1947, 2014.

[28] V. Acary and B. Brogliato, "Implicit Euler numerical simulations of sliding mode systems," Research Report RR-6886, Inria, 2009.

[29] A. Polyakov and A. Poznyak, "Reaching time estimation for "super-twisting" second order sliding mode controller via lyapunov function designing," IEEE Transactions on Automatic Control, vol. 54, no. 8, pp. 1951-1955, 2009.

[30] J. Moreno and A. Osorio, "Strict lyapunov functions for the super-twisting algorithm," IEEE Transactions on Automatic Control, vol. 57, no. 4, pp. 1035-1040, 2012. 